\title{
The Grate Italian Personality of the XXth Century - Pope John Paul I
}

\author{
Prof. Sergey Shkarovskiy \\ State University of Management, \\ High School of Economics, Moscow, Russia
}

\section{Doi:10.5901/ajis.2013.v2n9p544}

\begin{abstract}
The Pope John Paul I is an outstanding religious representative of the Catholic Church who significantly contributed to the Italian history as well as to the Christian world. Albino Luciani is remembered as «a smiling Pope». He eloquently demonstrated how to treat people, to love them and to respect human dignity. The brief biography of Albino Luciani is described. John Paul I was a highly educated man, he knew six foreign languages. Luciani showed his strong position concerning the reformism of the Catholic Church after participation in the IInd Vatican Council of 1962-1965. After the elections the new pope John Paul I began to carry out ecclesiastic reforms in order to bring the Church nearer to people. In this article the author shows difficult struggle Luciani had to sustain with the reactionary part of the Curia. John Paul I wanted to maintain discipline in the Church. He intended to continue fulfilling the commandments of Jesus Christ. Luciani had to be occupied with grave problems such as financial scandals in the Vatican Bank, abortion and use of contraceptives. The reasons for his elimination are analyzed. Prof. Shkarovskiy proved unnatural death of John Paul I and described the murder of the Pope. Albino Luciani is a saint person. It is proved by numerous miracles all over the world.
\end{abstract}

Keywords: Christianity, Catholic Church, reformism, ecclesiastic reforms, commandments, renovation, beatification, humanity.

Tra i rappresentanti religiosi di maggior rilievo spicca Papa Albino Luciani, che a seguito della sua elezione, scelse il nome di Giovanni Paolo I. Era il primo caso nella storia, in cui un potefice adottava il doppio nome. Ancora oggi questo papa è ricordato come «il papa del sorriso», perché ha conquistato il mondo con lo sguardo mite, l'onestà e l'umiltà.

Ebbe la capacità di accendere l'entusiasmo dei fedeli e di portare loro, gioia e serenità. Le sue prediche si distinguevano per una sincerità ed un calore umano che nessuno degli altri papi dell'età contemporanea ha mai avuto.

Giovanni Paolo I è sempre stato un Santo. Per sua intercessione, sono avvenuti molti miracoli in tutto il mondo.

Albino Luciani, futuro Giovanni Paolo I, è nato in un paese di montagna, all'epoca chiamato Forno di Canale (oggi Canale d'Agordo) nella provincia di Belluno in Veneto. Questo paese si trova nel cuore delle Dolomiti. La sua famiglia era povera e numerosa. Suo padre Giovanni Luciani si recava spesso all'estero in cerca di lavoro. La madre, Tankon Bortoli, era una donna molto religiosa, attendeva spesso e con pazienza il ritorno del marito dall'estero dove si recava per lavoro e si dedicava alla cura della famiglia. Durante gli anni della guerra e del primo dopoguerra, la famiglia Luciani patì notevolmente la fame.

Come accennato prima, la madre Bortole Tankon era una donna molto credente. Lei infondeva anche ai suoi bambini la fede. All'età di cinque anni, Albino cominciò a frequentare la chiesa, entrando a far parte del coro parrocchiale dei bambini. Col tempo partecipò sempre di più alle attività della fede cristiana, organizzate in parrocchia, con ardente entusiasmo di servire Dio e la gente, legando così il suo destino alla chiesa. II 17 ottobre del 1923, proprio nel giorno del suo undicesimo compleanno, Luciani lasciò la propria casa per recarsi prima a Belluno e poi a Feltre, per intraprendere gli studi in seminario.

Albino amava molto la lettura. La passione per la lettura risaliva ai tempi della prima infanzia, trascorsa in abbondante solitudine, in quanto non poteva giocare con i suoi coetani. All'età di cinque anni già leggeva M.Twain, H.Heine, J.Verne, J.Goethe, C.Dickens, C.Goldoni e molti altri scrittori e personaggi storici. Aveva una memoria fenomenale. Gli bastava una sola lettura per memorizzare tutto il contenuto;Gli apparteneva, inoltre, la particolare dote di ricordare tutte le date. Albino amava studiare ed era portato specialmente per le lingue, tanto da apprenderne ben cinque: latino, greco, francese, inglese e tedesco, oltre, ovviamente, alla conoscenza perfetta della lingua madre.

Nell'ottobre del 1928, Albino si è iscritto al Seminario Gregoriano di Belluno. Nel 1935, diviene diacono. In questo anno si verificano altri eventi: il 7 luglio viene nominato sacerdote nella chiesa di San Pietro a Belluno, dove il giorno dopo ha celebrato la sua prima messa. II 9 luglio viene nominato vicario, proprio nel paese d'origine, mentre il 18 dicembre intraprende il suo periodo di insegnamento presso l'istituto mineralogico. 
Nel periodo a seguire, Albino si iscrive alla Pontificia Università Gregoriana di Roma, alla facoltà di filosofia, con libertà di frequenza.

Nell'autunno del 1937, Don Luciani viene nominato prorettore dello stesso Seminario Gregoriano di Belluno, in cui si era formato.Luciani vi lavorerà per dieci anni, preparando i futuri sacerdoti e creando un'atmosfera molto familiare. Egli ricordava con piacere i tempi dei propri studi.

Aveva una spiccata capacità di attirare l'attenzione e l'interesse dei suoi studenti. La sua passione per l'arte, la storia, la religione e le scienze umanistiche gli ha permesso di trovare nuovi approcci all'insegnamento. Luciani inventava i giochi, scherzava, cercando di realizzare un'atmosfera rilassata e di sostegno in classe. ${ }^{1}$

Durante il periodo in cui Albino insegnava e svolgeva la sua missione pastorale, è scoppiata la Seconda Guerra Mondiale, un evento di fronte al quale non potè certamente essere indifferente, tanto da commentare con queste parole l'incontro del 19 luglio 1944 tra Mussolini e Hitler a Belluno: «Siamo nelle mani di due pazzi!»

Alla vigilia della nascita della Repubblica, e cioè il 23 novembre 1946, Albino discusse la tesi in filosofia presso la Pontificia Università Gregoriana, sul tema: «La natura dell'anima umana di Antonio Rosmini», un teologo italiano del XIX secolo, che nel 1848 scrisse un noto libro intitolato «Delle Cinque Piaghe della Santa Chiesa», che è stato immediatamente vietato dal Vaticano. In questa opera l'autore rileva la crisi della chiesa cattolica evidenziando cinque cause: 1) la distanza sociale del clero dal popolo; 2) il basso livello di formazione sacerdotale; 3) la dissociazione dei vescovi; 4) l'eccessivo interventismo della Chiesa nella vita sociale; 5) il concetto della proprietà e la dedizione della chiesa alla ricchezza. A causa di questo libro, Papa Pio IX tolse il cappello cardinalizio a Rosmini, avendo questa opera sconvolto profondamente il futuro pontefice.

In questo periodo, Albino Luciani intraprese anche la sua attività giornalistica, cominciando a collaborare con il giornale: «L'Amico del Popolo» su cui scrisse svariati articoli pacifisti, ispirato dal movimento giovanile della Democrazia Cristiana.

I capi locali della chiesa cominciarono a prestare attenzione per il giovane e virtuoso Don Luciani, che da quel momento iniziò l'ascesa alla scala gerarchica della chiesa, tanto da passare in soli 6 anni da vicario ordinario (02.02.1946) a vicario generale della diocesi di Belluno (06.02.1954).

Sotto questa nuova veste avrà modo di ospitare presso la propria vicaria il patriarca di Venezia Angelo Roncalli, futuro Papa Giovanni XXIII-secondo (Giovanni XXIII-primo, Baldassarre Cossa, che aveva regnato la Chiesa nel periodo 1410-1415, attirandosi l'appellativo di antipapa, oggi è sepolto nel Battistero di Firenze, città in cui è tutt'oggi venerato dai fiorentini), in occasione del Congresso Eucaristico da lui stesso organizzato. II 27 dicembre 1958, in Piazza San Pietro a Roma, sono stati nominati da Giovanni XXIII-secondo, otto nuovi vescovi, tra cui Mons. Luciani.

Luciani amava parlare con la gente, andare a trovare le persone malate, conversare con i bambini durante le prediche. Frequentava tutte le sue parrocchie e amava ascoltare i problemi dei vari parrocchiani per aiutarli. Quando un bambino ammalato doveva ricevere la cresima, Albino si recava personalmente a casa sua.

Sebbene Luciani, in qualità di vescovo, potesse delegare molte delle sue funzioni, in realtà impegnò il meno possibile i suoi collaboratori. Luciani non amava sfoggiare gli abiti da alto prelato, in quanto preferiva vestire come un semplice sacerdote e, soltanto nelle occasioni formali, indossava la veste da vescovo.

L'umiltà di Albino Luciani non deve tuttavia essere interpretata, come un fatto di debolezza, poichè quando bisognava affrontare le questioni difficili, egli sapeva essere fermo e deciso e farsi rispettare. ${ }^{2}$

Gli anni 60 furono per la chiesa cattolica un periodo di grandi cambiamenti. Giovanni XXIII-secondo aveva capito che era sopravvenuto il tempo di riformare la chiesa, rimasta troppo indietro rispetto all'evolversi dei tempi. La chiesa, in pratica, non era più al passo con i tempi. Convocò, a tal fine, il Concilio Vaticano II nell'anno 1962 che durò fino al 1965, sotto il suo successore Paolo VI. Nel corso del concilio furono approvati i documenti fondamentali per la vita della chiesa: quattro costituzioni, nove decreti e tre dichiarazioni. 2.540 vescovi cattolici parteciparono a questo evento epocale.

Albino Luciani ha attivamente partecipato al Secondo Concilio Vaticano. Luciani aderiì energicamente alle nuove idee del Consiglio, cui rimase fedele per tutta la vita.

Nel 1969 morì il patriarca di Venezia Urbani. Paolo VI, decise di far occupare la sede vacante proprio dal vescovo di Vittorio Veneto: Albino Luciani. II 15 dicembre dello stesso anno. Mons. Luciani venne nominato Patriarca di Venezia.

L'unica ricchezza, che Luciani, si portò dietro da Vittorio Veneto, era l'imponente mole di libri. Luciani non spese un soldo nemmeno per farsi le nuove vesti cardinalizie, in quanto utilizzò quelle già usate dal suo predecessore. Girava per

\footnotetext{
${ }^{1}$ Serafini L. Albino Luciani II papa del sorriso, Messaggiero di Sant'Antonio, Padova, 2008, 186pp.

2 Incitti L. Papa Luciani. Una morte sospetta, L'Airone Editrice, Roma, 2001, 125pp. 
Venezia, vestito con semplice abito da prete, zucchetto e croce pettorale arrotolati in tasca. Dai primi giorni del suo patriarcato, Albino Luciani impartì l'ordine di lasciare aperte le porte del patriarcato a tutte le ore del giorno per i fedeli. Non fece mai eccezione per nessuno, accogliendo sempre tutti i visitatori.

Come patriarca Albino ricordò sempre la centralità del catechismo. Nel corso delle sue prediche, non esitava mai ad affrontare i problemi più ostici e vitali, che affliggevano i veneziani e cercava di calmarne gli animi.

In Italia, nel 1974, venne attivamente discussa la legge per l'approvazione del divorzio. In quell'occasione fu indetto un referendum. Albino Luciani invitò i sacerdoti ed i fedeli a conformarsi alla dottrina ufficiale della Chiesa, 0 almeno a non parlare apertamente a favore del divorzio. L'associazione universitaria $\mathrm{FUCl}$ pubblicò un opuscolo «Riflessioni pastorali e politiche in occasione del referendum», in cui si parlò apertamente a favore del divorzio. ॥ patriarca di Venezia volle sciogliere questa organizzazione giovanile. La risposta non tardò a venire. Gli studenti accusarono Luciani di essere troppo chiuso e conservatore.

Nel 1972, in occasione di una visita pastorale di Paolo VI a Udine per il Congresso Eucaristico Nazionale, nella giornata del 16 settembre, Papa Montini decise di accompagnare il Patriarca Luciani a Venezia, fino a Piazza San Marco. Alla cerimonia sopraggiunsero ben 20.000 persone. II Papa salutò tutti in compagnia del Patriarca sorridente. Al termine della cerimonia, Paolo VI si tolse imporvvisamente la stola per porla sulle spalle di Luciani, cogliendo tutti di sorpresa.

Albino Luciani stesso, racconterà di quel giorno, nell'Angelus di domenica 27 agosto 1978, il giorno successivo della sua elezione a Sommo Ponfice. Paolo VI con questo gesto simbolico ha dimostrato a tutti che lo avrebbe preferito come suo successore.

Paolo VI pensò anche all'opportunità di aprire un nuovo concistoro. II 5 marzo 1973, monsignor Luciani, insieme al patriarca di Lisbona e ad altri nuovi cardinali, riceve la berretta cardinalizia direttamente dalle mani del pontefice. In quell'occasione toccò a lui leggere il discorso a nome dei nuovi cardinali, in quanto l'anno precedente era stato eletto vice-presidente della Conferenza Episcopale Italiana.

In qualità di cardinale, Luciani ha effettuato quattro visite pastorali fuori d'Italia. II suo primo viaggio fu in Svizzera, nel 1971, dove incontrò i lavoratori immigrati italiani nella città di Einsielden. Nel maggio del 1975, si recò alla città tedesca di Mainz, per festeggiare il millenario dalla realizzazione del duomo, incontrando anche in quella circostanza gli immigrati italiani ed il cardinale Folk, che ha ritrovato in conclave nell'agosto del 1978. Nello stesso 1975, ha fatto una visita ufficiale in Brasile, dove gli venne conferita la laurea: "honoris causa" dall'università di Santa Maria a Rio Grande do Sul. In Brasile, Luciani ebbe modo di conoscere un altro grande cardinale Aloisio Lorscheider, con cui strinse un'amicizia così forte, da votarlo persino, nel conclave del 1978.

Papa Luciani fece il suo quarto ed ultimo viaggio, nel 1976, questa volta in Jugoslavia, dove fu accolto nella città di Split in occasione del millenario del primo santuario, in qualità di rappresentante ufficiale della CEI (Conferenza episcopale italiana).

Albino Luciani conosceva molte lingue straniere. Lo studio delle lingue era una delle sue maggiori passioni, insieme al giornalismo. Parlava benissimo: latino, tedesco, francese, spagnolo e inglese. Nel 1976, incontrò un giovane prete, Diego Lorenzi, che diventò successivamente il suo segretario in Vaticano.

Albino Luciani amava scrivere. Nel 1976 a Padova, fece pubblicare dalla casa editrice «Messaggero di Sant'Antonio» un libro intitolato «lllustrissimi», che a seguito della sua elezione pontificale, diventò un best-seller. In questo libro Albino Luciani dialoga con 40 personaggi illustri e famosi, quali: Gesù Cristo, Francesco Petrarca, Pinocchio, Maria Teresa d'Austria, re David, Charles Dickens, Cicikov, Figaro, Goethe, Carlo Goldoni, Ippocrate, Sir Walter Scott e Mark Twain. In tal modo originale, Luciani ha espresso il suo punto di vista e tutti i suoi pensieri sugli eventi attuali che interessavano il paese ed il mondo, sulle sfide future della Chiesa. ${ }^{3}$

Nel luglio del 1977 cardinale Luciani guidò un pellegrinaggio pastorale alla città portoghese di Fatima. Questa città è famosa nel mondo per le apparizioni della Madonna, a cominciare dal lontano 13 maggio 1917, a tre pastorelli, tra cui fu Lucia Dos Santos, Lei diventò suora. Suor Lucia è morta il 13 febbraio del 2005. .

L'11 luglio del 1977, suor Lucia incotrò cardinale Luciani. La cosa sconvolgente di questo memorabile incontro, fu, che la stessa suor Lucia salutò Luciani con l'appellativo di «Santo Padre». La veggente, caduta in uno stato di trans, disse che al patriarca lo avrebbe atteso la corona di Cristo ed un breve pontificato.

Il 6 agosto 1978, Papa Paolo VI morì a Castel Gandolfo, la residenza estiva fuori Roma. Iniziò, così, il periodo di «sede vacante». II cardinale Luciani con il suo segretario don Diego Lofrenzi partì per il conclave. 
II 1 ottobre 1975, Paolo VI, sapendo che avrebbe lasciato a breve questo mondo, pubblicò l'enciclica, in cui stabili una nuova procedura per l'elezione del successore di Pietro, regolamentando il periodo della «sede vacante». Stabili, in particolare, che tutti i cadinali, dovessero rassegnare le dimissioni, per dare al nuovo papa piena libertà di fare nuove nomine. II posto di camerlengo sarebbe spettato al Segretario di Stato Jean-Marie Villot. La gestione generale della Chiesa sarebbe stata svolta dal Sacro Collegio dei Cardinali, che fissò l'inizio del conclave per il 25 agosto. Al conclave di fine agosto parteciparono 111 cardianli.

Secondo i mass media, ancora una volta, il futuro papa sarebbe stato un italiano. Ma non tutti i cardinali condividevano questo punto di vista, in particolar modo i cardinali non italiani. Anche il cardinale Luciani desiderava che il nuovo papa fosse il rappresentante dell'America Latina. Egli, infatti, appoggiava apertamente il cardinale brasiliano Aloisio Lorscheider, che conosceva bene. Albino Luciani, il 10 agosto del 1978, dichiarò apertamente alla sua Diocesi, che avrebbe votato per il cardinale brasiliano.

Si sentiva il bisogno di un papa pastore, gran devoto, favorevole alla divisione dei poteri ed alla collegialità, in grado di riconoscere i reali bisogni della gente. Bisognava promuovere la collaborazione tra i paesi occidentali ed orientali (NATO e Patto Varsavia).

Oltre ai problemi di carattere mondiale, vi erano i problemi interni dell'Italia, in particolare, attinenti ai rapporti tra cattolici e comunisti. Proprio in quegli anni '70, l'Italia dovette affrontare il peggior periodo dal dopoguerra: il terrorismo delle «brigate rosse». Questa piaga sociale ha ricevuto il suo picco nei mesi marzo-maggio, quando fu rapito e poi assassinato il primo ministro Aldo Moro.

Incombevano al futuro pontefice i più gravi problemi, sorti sotto il pontificato di Paolo VI, quali: I'organizzazione interna della Chiesa, l'evangelizzazione, l'ecumenismo, la collegialità ecclesiastica, il ruolo dei vescovi, l'equilibrio tra il modernismo e tradizionalismo, il controllo delle nascite, il celibato obbligatorio del clero, il ruolo delle donne nella vita della Chiesa, la teologia, la libertà e la lotta per la pace nel mondo. Particolarmente acuto era il problema del risanamento delle finanze vaticane, soprattutto a seguito del clamoroso scandalo del fallimento del «Banco Ambrosiano», con le conseguenti ripercussioni sulle finanze della Banca Vaticana: Io IOR, guidato da Mons. Marcincus.

La Curia Romana voleva scegliere il proprio candidato tra i cardinali che intendessero proseguire l'opera intrapresa dai predecessori.

Il nome del Patriarca di Venezia, Albino Luciani non fu menzionato, inizialmente, da alcuna testata giornalistica. Ma ciò non significò assolutamente che la sua candidatura non venisse presa in considerazione, tanto che la maggior parte dei cardinali dell'America Latina propose proprio Luciani come il successore di Pietro. Di gran rilievo per l'elezione di Luciani al soglio pontificio, fu soprattutto il contributo del cardinale di Firenze, Benelli.

Poco tempo prima del conclave, il nome di Luciani fu menzionato soltanto da un quotidiano italiano «ll Giorno» e da uno francese «Le Manin». II 22 agosto il giornalista italiano Gianfranco Zizola ha pubblicato nel quotidiano «॥ Giorno» un articolo intitolato «Chi sarà il nuovo papa? Albino Luciani. Con i poveri (ma non a sinistra)». ${ }^{4}$ I giorno dopo "Le Manin» di Parigi ha ricopiato lo stesso articolo in francese. L'opinione di Zizola si basò sull'intervista concessa dal cardinal Benelli poco tempo prima del Conclave, nonché sulle informazioni ricevute da una delle coalizioni cardinalizie che avevano considerato Luciani uno dei nominabili. Questo gruppo era tuttavia fortemente osteggiato dal conservatore Siri. ${ }^{5}$

Fu allora che i giornalisti cominciarono subito ad intervistare il patriarca di Venezia. A loro, Luciani, rispose dicendo di essere al massimo nella lista di serie $\mathrm{C}$ per la nomina papale» ed il suo nome fu rapidamente «dimenticato».

II 25 agosto, proprio prima del conclave, lo stesso «Le Manin» ha pronosticato nell'articolo «Les dernières manoeuvres vaticanes» («Le ultime manovre vaticane») la candidatura di Luciani, sostenuta dai cardinali africani e latino americani, nonché dai pochi cardinali curiali, inclusi Pellegrino ed Ursi. Nell'edizione pomeridiana il giornale francese ha aggiunto che l'alleanza si aprì anche ai conservatori, perché nella biografia di Luciani, si constatarono anche episodi di fermezza e di restaurazione dottrinale e disciplinare. Alla fine, il 27 agosto «Le Manin», in attesa dei risultati del conclave, scrisse che Luciani aveva le più forti chances di conseguire l'elezione papale all'unanimità, rispetto ad altri Cardinali più estremisti come Siri e Benelli. Luciani era assolutamente sicuro di non essere eletto.

|| 26 agosto di mattina I cardinali hanno celebrato la messa «per l'elezione di un pontefice romano», sono entrati nella Capella Sistina. Cardinale Luciani è stato eletto Papa alle 18.05 dello stesso giorno dopo il quarto scrutinio. Lui ha scelto il nome Giovanni Paolo I, il primo doppio nome nella storia della Chiesa cattolica.

\footnotetext{
${ }^{4}$ Chi sarà il nuovo papa? Albino Luciani. Con i poveri (non a sinistra), «ll Giorno», 22 agosto 1978.

5 Un papabile de compromis, Albino Luciani, «Le Manin» (Paris), 23 agosto 1978.
} 
Alle 19:18 si sono aperte le finestre sul balcone della Basilica San Pietro. Si è affacciato lo stesso Luciani un po'stanco, sebbene si vedessero bene sul suo viso la gioia ed il sorriso, che hanno colpito subito il mondo intero.

II 27 agosto all'ultima riunione del conclave nella Cappella Sistina Giovanni Paolo I fece il suo primo discorso ufficiale davanti a tutti i cardinali. Luciani proclamò il suo programma, che includeva 6 argomenti fondamentali:

1. «Vogliamo continuare nella prosecuzione dell'eredità del Consiglio Vaticano II, vegliando affinchè non se ne travisino i contenuti ed i significati, e non se ne rallenti il magnifico impulso di rinnovamento».

2. «Vogliamo conservare intatta la grande disciplina della Chiesa, nella vita dei sacerdoti e dei fedeli, sia nell'esercizio delle virtù evangeliche che nel servizio dei poveri, degli umili e degli indifesi»».

3. «Vogliamo ricordare alla Chiesa intera che il suo primo dovere resta sempre quello della evangelizzazione».

4. «Vogliamo continuare lo sforzo ecumenico, vegliando con fede immutata, con speranza intatta e con amore indeclinabile, alla realizzazione del grande comandamento di Cristo: essere una cosa sola».

5. «Vogliamo proseguire con pazienza e fermezza in quel dialogo sereno e costruttivo, che il compianto Paolo $\mathrm{VI}$ ha posto a fondamento e programma della sua azione».

6. «Vogliamo infine favorire tutte le inziative lodevoli e buone che possano tutelare ed incrementare la pace nel mondo». ${ }^{6}$

Quasi subito dopo l'elezione a papa iniziò a lavorare per una nuova enciclica, da dedicare all'unità della chiesa, alle donne, alla collegialità ed alla povertà.

Lo stesso giorno, a mezzogiorno, il nuovo pontefice ha tenuto il suo primo Angelus domenicale davanti a duecento mila persone raccolte in piazza San Pietro. Milioni di persone da tutto il mondo, guardavano ed ascoltavano, in diretta, il loro nuovo vicario di Cristo. Luciani, prima di recitare la preghiera, si rivolse spontaneamente ai fedeli accorsi nella gran piazza, con linguaggio semplice e comprensibile a tutti, dicendo:

«leri, ieri mattina, son andato alla Sistina a votare tranquillamente. Mai avrei immaginato quello che stava per succedere. Appena comiciato il pericolo per me, i due colleghi che erano vicini, mi hanno sussurato parole di coraggio. Uno ha detto: «Coraggio: se il Signore dà un peso, dà anche l'aiuto per portarlo». E l'altro collega: «Non abbia paura, in tutto il mondo c'è tanta, tanta gente, che prega per il papa nuovo. Venuto il momento ho accettato. Spero che mi aiuterete con le vostre preghiere».

Nessuno si aspettava un discorso così tanto alla mano. Un'altra cosa che ha colpito tutti è il sorriso di Albino Luciani. Il suo discorso venne interrotto svariate volte, da applausi ed acclamazioni. La gente era impressionata dalla sua umiltà. II Papa ha conquistato le anime di tutte le persone che lo sentivano e lo guardavano. Papa Luciani aveva il dono di Dio, di saper contagiare la gente con la sua energia positiva. Ancora una volta, impressionò tutti con il suo sorriso. Da subito lo chiamarono «Papa del sorriso»; nessuno si aspettava da lui di sentirlo parlare in prima persona, rinunciando al plurale maiestatis. Le sue parole penetrarono nell'animo di ogni fedele.

Giovanni Paolo I decise di semplificare notevolmente la tradizionale cerimonia d'inizio del pontificato. A questo evento fu tolto il nome di «messa d'incoronazione», sostituito con quello di «intronizzazione». Luciani ha abolito l'uso della tiara, il prezioso triregno a forma di ogiva sormontato da tre corone e la sedia gestatoria. II Papa salirà a piedi all'altare. La cerimonia non durerà più di 6 ore. Per ottenere questo risultato, dovette sopportare un lungo dibattito nel corso del quale rimase irremovibile. Lo stesso giorno Giovanni Paolo I lasciò Villot nella posizione di Segretario di Stato e riconfermò le cariche degli altri cardinali in Curia.

Nella giornata di venerdì 1 settembre 1978, il papa tenne la sua prima conferenza stampa con i giornalisti. In tale occasione rivelò pienamente il suo talento giornalistico. Alla riunione parteciparono 300 persone. Giovanni Paolo I, confidò loro che se non fosse diventato prete, avrebbe fatto il giornalista.

I| 5 settembre, Giovanni Paolo I ricevette la delegazione delle confessioni cristiane non cattoliche che parteciparono alla cerimonia di intronizzazione del 3 settembre. Tra gli inviati ci furono i rappresentanti della Chiesa ortodossa russa. La delegazione russa fu guidata dal metropolita di Leningrado Nikodim (Boris Rotov). Alle 10:00 Nikodim mentre presentava i membri della sua delegazione, improvvisamente, cadde tra le braccia del pontefice. ॥ Metropolita venne colto da malore mortale: infarto per arresto cardiaco. Nikodim stimava molto il cattolicesimo e desiderava riavvicinare le due chiese sorelle. Pochi anni dopo, venne, persino, avanzata un'ipotesi mai confermata, che il Metropolita morì avvelenato per aver bevuto nel calice, presumibilmente destinato a Giovanni Paolo I. Nikodim aveva solo 49 anni. $^{7}$

\footnotetext{
${ }^{6}$ Albino Luciani dal Veneto al mondo, Viella, Vicenza, 2010, 602pp.

${ }^{7}$ http://www.religio.ru/lecsicon/03/120.html
} 
Giovanni Paolo I durante il suo breve pontificato ha avuto quattro Udienze generali con i suoi fedeli nella grande Sala Nervi del Vaticano, che accoglieva circa 15.000 fedeli. II primo di questi eventi ha avuto luogo il 6 settembre. II tema della riunione era «La grande virtù dell'umiltà».

Domenica, 10 settembre dopo aver celebrato la messa mattutina, alle ore 12,00 a.m. si è affacciato alla finestra del terzo piano del Palazzo Apostolico per recitare la tradizionale preghiera domenicale dell'Angelus Domini, poi si è soffermato sulla necessità di risolvere il conflitto nel Medio Oriente, citando il Vecchio ed il Nuovo Testamento e persino il Corano. Alla fine della preghiera disse:

«Noi siamo oggetto da parte di Dio di un amore intramontabile. Sappiamo che ha sempre gli occhi su di noi, anche quando sembra che ci sia solo la notte. È papà; più ancora è madre!»

Queste parole hanno spaventato subito i nemici di Albino Luciani, che, in risposta alle loro critiche, disse che tali parole erano state dette dal profeta Isaia.

La giornata del 12 settembre fu molto drammatica. Sulla scrivania del Papa comparve il numero appena uscito della rivista OP ("Osservatore Politico»), del giornalista Mino Pecorelli, che pubblicò l'elenco dei membri della loggia massonica P2, che includeva 131 nomi di persone religiose di alto livello, con tanto di numero di registrazione e soprannome, all'interno dell'organizzazione massonica.

Tra i «pezzi grossi» erano comparsi i nomi di Villot, Casaroli, Marcincus, Levy (vice direttore del quotidiano vaticano "Osservatore Romano»), Tucci (direttore di Radio Vaticana). Per Luciani fu l'ennesima piaga da risolvere, sebbene avesse già sentito queste voci. II Papa aveva il potere di scomunicare queste figure dalla chiesa. Lo stesso Pecorelli era stato anche lui membro della loggia, ma ne fu deluso e per questo iniziò a contrastarla. Questa azione coraggiosa gli è costata però, la vita. Morì, infatti, assassinato il 20 marzo 1979 a Roma.

Nella giornata del 13 settembre, Giovanni Paolo I iniziò a studiare la situazione dello IOR. II pontefice si preparò ad incontrare i rappresentanti degli Stati Uniti, che volevano discutere con lui delle diverse operazioni finanziarie illecite, fatte da Sindona, Calvi e Marcincus. All'incontro con il pontefice, vennero sei persone: due uditori della Banca d'Italia, e quattro rappresentanti della FBI (Federal Bureau of Investigation degli Stati Uniti, Ufficio federale di investigazione) e del ministero di giustizia americano che comunicarono al Papa di aver preparato una relazione sulle frodi finanziarie di Marcincus, Sindona e Calvi, e che prima o poi tutto ciò sarebbe stato reso pubblico, preannunciando che a seguito di questo fatto, la Santa Sede sarebbe stata colpita da un gravissimo scandalo. Giovanni Paolo I sapeva già bene come avrebbe dovuto agire, ma il tempo disponibile era ormai poco. ${ }^{8}$

Al suo secondo incontro nella Sala Nervi, erano presenti quasi ventimila persone e la sala era totalmente piena. La Curia romana convinse il Papa a sedersi sulla sedia gestatoria affinchè tutti i presenti avessero la possibilità di vederlo. Giovanni Paolo I, parlò della fede, citando il poeta romano Trilussa.

II 19 settembre, Giovanni Paolo I iniziò ad organizzare un piano di sostituzioni da apportare in Vaticano ed a preparare nuove encicliche ringuardanti i futuri cambiamenti della Chiesa cattolica, secondo gli insegnamenti del suo fondatore Gesù Cristo, perché sentiva la necessità di riportare la Chiesa alle origini, e quindi a maggiore semplicità, umiltà e vicinanza a Dio ed ai fedeli.

Martedì il 20 settembre, continuò a dedicarsi al rinnovamento della chiesa. Nella stessa sera, lo attendeva la sua terza udienza con i fedeli alla Sala Nervi. Dedicò questo terzo incontro con i cattolici, alla virtù teologale della speranza.

Sabato il 22 settembre Papa Luciani fece una sola visita fuori il Vaticano. si preparò per la sua prima e sola visita ufficiale fuori del Vaticano, nella Basilica di San Giovanni in Laterano. II Papa dovette cedere per la seconda volta ai cardinali della curia romana e sedersi sulla sedia gestatoria, che lo portò sino all'entrata della Basilica. In quel giorno, per l'ultima volta venne usata la sedia gestatoria, che il papa successivo ripose, in seguito, nei Musei Vaticani.

Martedì il 26 il pontefice completò la preparazione del nuovo organigramma e la dottrina aggiornata della Chiesa. Quella stessa sera, si svolse l'ultima udienza pubblica tenuta da papa Luciani nella Sala Nervi. II tema di quell'incontro fu la carità.

II 28 settembre fu l'ultimo giorno di vita di Giovanni Paolo I. Quella mattina Albino Luciani si alzò alle 4:30. Alle 7:00 fece colazione ed alle 8:00 iniziò a lavorare.

II Papa contattò i cardinali Pericle Felici e Giovanni Benelli. Luciani ebbe un colloquio con il cardinale Baggio, cui comunicò la decisione di sostituire il cardinale Cody di Chicago (arcivescovo di Chicago, una della più ricche diocesi del mondo. La sua diocesi comprendeva 2,5 milioni di fedeli, più di tremila sacerdoti ed oltre 450 parrocchie. Cody aveva, infatti, occultato le entrate economiche diocesane, facendole proprie, per un ammontare di circa 250 milioni di dollari). 
Baggio accolse questa decisione che sperava da tempo. Preannunciò, successivamente, allo stesso, la nomina a patriarca di Venezia, che rimase sede vacante. II cardinale Baggio, tuttavia, si rifiutò. Luciani né rimase scioccato, in quanto abituato da sempre alla disciplina della Chiesa: I'obbedienza al Papa e l'esecuzione incondizionata dei suoi ordini erano, infatti, concetti da lui assimilati sin dai tempi del seminario.

Verso sera, ebbe luogo, una lunga ed accesa conversazione con Villot. La prima cosa che il Papa gli disse era che intendeva rimuovere al più presto Marcincus dalla posizione di direzione della Banca Vaticana, per affidarne il posto a Giovanni Gobbo, Segretario della Prefettura per gli Affari Economici della Santa Sede e nominare Marcinkus vescovo ausiliare di Chicago. In seguito, il papa disse di voler chiedere le dimissioni del cardinal Cody di Chicago. Luciani, inoltre, riferì circa il rifiuto di Baggio di guidare il Patriarcato di Venezia. In seguito, Giovanni Paolo I informò il cardinale Villot degli ulteriori cambiamenti che aveva in programma. II cardinal Felici sarebbe divenuto vicario di Roma, al posto di Poletti, mentre lo stesso Villot sarebbe stato sostituito dal cardinale Benelli, come Segretario di Stato. Quest'ultima decisione non piacque a Villot. Questa conversazione durò quasi due ore.

Dopo l'accesa conversazione con il Segretario di Stato in carica Villot, il pontefice parlò telefonicamente con il cardinale Colombo, che subito dopo la morte del pontefice, disse:

«Giovanni Paolo I mi ha parlato a lungo con tono normalissimo, dal quale non era possibile arguire qualsiasi malore fisico. II saluto finale invocava preghiere, ed era pieno di serenità e speranza».

Alle 21:30, Papa Giovanni Paolo I si congedò con queste ultime parole ai suoi segretari: «Buona notte. A domani. Se Dio vuole». ${ }^{9}$

Poi chiuse la porta. La mattina seguente il pontefice verrà trovato morto.

Venerdì mattina il 29 settembre 1978, suor Vincenza Taffarel ha scoperto il corpo senza vita di Luciani. Alle 4:30 lei ha portato, come ogni mattina, la caffettiera nello studio del Papa. Lo ha salutato, ma non ha ricevuto stranamente risposta. Dopo un quarto d'ora è rientrata nello studio, ed ha notato la caffetteria ancora intatta. Di solito Papa Luciani prendeva subito il caffè. Per questo motivo, Suor Vincenza si è decisa a bussare direttamente alla porta della camera da letto del papa. Ha iniziato a bussare da piano a sempre più forte. Nessuna risposta. Allora ha deciso di fare ciò che non aveva mai osato prima: entrare nella stanza papale. L'attendeva una scena terribile. Albino Luciani morto sul letto appoggiato allo schienale. La sua testa era inclinata lievemente a destra mentre gli occhi erano aperti. Pare che Giovanni Paolo I tenesse in mano degli appunti con il nuovo organigramma del Vaticano. La luce era accesa. Suor Vincenza si è avvicinata al Papa e ha sentito il polso. La mano era ancora tiepida, ma non c'era nessun segno di vita. Giovanni Paolo I era già morto, per cui resasi immediatamente conto dell'accaduto, è corsa via incredula, spaventata ed in lagrime per andare a chiamare don Diego Lorenzi, che Albino Luciani aveva preso con se insieme a lei in Vaticano. L'appartamento di Don Diego era accanto a quello papale. Poi, si è precipitata a chiamare l'altro segretario: don Magee. Don Lorenzi è rimasto in stato di choc, a vedere il corpo del papa senza vita. In soli due mesi don Magee ha visto ben due papi defunti. Ma qual'era la differenza ? II 6 agosto a Castel Gandolfo muore Papa Paolo VI, circondato da tanta gente. Mentre la morte di Giovanni Paolo Primo è avvenuta in totale solitudine.

Gli eventi successivi rimangono ancor più inspiegabili. Intorno alle 5:00, il cardinal Villot entra nell'appartamento del papa appena morto: si affretta a far sparire subito tutti gli oggetti personali del papa: occhiali, scarpe, medicine, appunti, e quant'altro si trovasse nella sua camera da letto. Dopo sole 12 ore dalla scoperta del papa deceduto non c'èra già più nessuna traccia di Albino Luciani pontefice romano.

Villot ha chiamato Buzzonetti, il medico del Vaticano, che ha fatto un esame esterno del corpo di Luciani. II medico ha diagnosticato un infarto del miocardio. Ci si chiede se si possa fare una valida diagnosi senza autopsia! II medico ha inoltre costatato che la morte sarebbe avvenuta intorno alle 23.00 del giorno precedente, per l'appunto del 28 settembre. $\mathrm{Ma}$ anche questo dato si sarebbe potuto accertare con maggiore precisione col predetto esame che in realtà non è stato mai effettuato. Buzzonetti ha, inoltre, conosciuo il papa solo sul letto di morte, non avendolo mai né incontrato né visitato prima. L'assenza dell'autopsia e la discordanza delle sue conclusioni con altre risultanze, né hanno comportato un'inevitabile giudizio di presunzione. II medico della Santa Sede, non sapeva nemmeno quali medicine prendesse il Papa. Inoltre, il documento ufficiale sulla morte del Santo Padre è stato pubblicato addirittura ad una sola settimana dalla morte. Tutto questo evidenzia come le cause della morte di Giovanni Paolo I possano essere, con ogni probabilità, del tutto innaturali.

\footnotetext{
9 Yallop D., In God's name, BANTAM BOOKS, London, 1984, 388pp.
} 
In quella stessa mattina, Villot ha chiamato i fratelli Signoracci al fine di far imbalsamare il corpo del papa morto. Si trattava per gli stessi della terza chiamata, avendo imbalsamato già altri due papi prima di lui: (Giovanni XXIII-secondo nel giugno del 1963 e Paolo VI nell'agosto del 1978). Un'auto del Vaticano è stata così inviata per andarli a prendere.

Altro fatto strano, che merita considerazione, è che in quella stessa mattina, verso le 6:45, Marcincus si avvicinanò al Vaticano, cosa del tutto anomala se si considera che in 30 anni non si era mai alzato presto.

Appena saputo della morte del pontefice, Villot ha intimato a tutti di tacere. Nonostante il divieto, Don Lorenzi informa ugualmente il medico personale di Luciani: Giuseppe Da Rosa che curava Luciani da più di 20 anni e che si trovava in quel momento a Venezia. Da Rosa, saputo della morte del pontefice, si è recato immediatamente a Roma, senza fare in tempo a vedere il corpo del papa, prima dell'imbalsamazione.

Don Diego Lorenzi ha chiamato successivamente Pia Luciani, la nipote più amata del Papa.

Alle 6:30 il telegiornale straordinario su RAIUNO ha trasmesso la notizia sulla morte di Giovanni Paolo I. Alle 7:20 suonavano le campane funebri di Canale D'Agordo, paese natio di Albino Luciani. Alle 7:27 Radio Vaticana trasmetteva il testo scritto dal cardinale Villot, diventato automaticamente camerlengo. Ecco il comunicato fatto trasmettere dal cardinale «grigio»:

«Questa mattina, 29 settembre 1978, verso le cinque e mezzo, il segretario privato del Papa, non avendo trovato, diversamente dal solito, il Santo Padre nella cappella del suo appartamento privato. Lo ha cercato nella sua stanza e lo ha trovato morto nel letto con la luce accesa, come se fosse intento a leggere. II medico, dottor Renato Buzzonetti, che è accorso nella stanza del Papa, ha confermato la morte, che è avvenuta presumibilmente verso le undici di ieri sera, asserendo che si è trattato di «morte improvvisa, che potrebbe essere stata causata da infarto miocardico acuto». ${ }^{10}$

Questo messaggio rivela, tuttavia, nonostante la sua brevità, tre discordanze. In primo luogo, che il Papa è stato trovato morto alle 4:45 anziché alle 5:30. In secondo luogo, che è stata suor Vincenza a scoprire il cadavere del papa e non il segretario privato. In terzo luogo che il papa non poteva essere morto alle 23:00 del 28 settembre, perché quando suor Vincenza ha verificato il polso del papa, la mano era ancora tiepida.

A questo punto sorge una domanda - perché Villot abbia fatto diffondere quel comunicato ufficiale così falso ? Più tardi, Villot ha corretto il suo comunicato. II segreratio che avrebbe scoperto il papa morto sarebbe stato don Magee, un'altra bugia. Secondo Villot, Giovanni Paolo I non teneva in mano un foglio con il nuovo organigramma del Vaticano, ma una copia del libro «Le imitazione di Cristo». Anche questa informazione era falsa, perché il libro del Papa si trovava ancora a Venezia. Alcuni giorni prima, poiché Luciani desiderava trarre alcune citazioni dal libro, aveva inviato don Lorenzi a prenderne in prestito una copia dal suo confessore. Tuttavia, lo stesso, era stato già restituito al proprietario qualche giorno prima che il papa morisse. II Vaticano sotto la direzione di Villot continuava a disorientare tutto il mondo fino al 2 ottobre.

Sempre il cardinal Villot ha preso la decisione di imbalsamare il corpo del Papa molto precipitosamente, superando la dura resistenza di due cardinali di spicco: Felice e Benelli, al corrente del nuovo organigramma vaticano che Luciani voleva pubblicare proprio il 29 settembre. Dal mondo esterno alle mura vaticane, si facevano sempre più accese le richieste di sottoporre il corpo del papa ad autopsia. Secondo le leggi italiane i corpi dei defunti possono essere imbalsamati dopo 24 ore dalla morte. II corpo di Paolo VI è stato imbalsamato in conformità con le leggi italiane. Secondo il diritto canonico, il camerlengo ha il potere di vietare l'autopsia e Villot naturalmente se ne è avvalso. L'ultima autopsia in Vaticano era stata eseguita nel 1830 sul corpo di Pio VIII.

Lo stesso giorno per la prima volta, il corpo di Giovanni Paolo I è stato esposto al pubblico nella sala Clementina accanto agli appartamenti papali. II corpo del Papa non era ancora imbalsamato. Alle 11:00 l'hanno portato via. II processo di imbalsamazione è iniziato sotto controllo. I fratelli Signoracci hanno ricevuto l'ordine di non estrarre niente dal corpo. Perché è stata data questa disposizione? Evidentemente per far sparire le tracce del reato. Del resto in che altro modo si sarebbe potuto scoprire la verità ?

Ultimo elemento sbalorditivo è che i fratelli Signoracci dopo aver esaminato il corpo del papa, hanno constatato che Albino Luciani non poteva che essere morto tra le 4:00 e 5:00 del 29 settembre e non alle 23:00 del 28 settembre. Questo significa che suor Vincenza era entrata negli appartamenti papali quasi subito dopo la morte. Solo l'autopsia poteva dissipare tutti i dubbi e reprimere ogni controversia direttamente sul nascere. L'imbalsamazione è stata completata nel pomeriggio del 30 settembre.

Ci sono altri fatti che confermano la versione dell'omicidio di Giovanni Paolo I. Perché l'autovettura del Vaticano che doveva andare a prendere i fratelli Signoracci è partita alle 5:00 a.m. e cioè prima ancora che il corpo di Luciani

\footnotetext{
${ }^{10}$ Yallop D., In God's name, BANTAM BOOKS, London, 1984, 388pp.
} 
venisse trovato morto alle 5:30 a.m. nella sua stanza ? Probabilmente Villot era già a conoscenza della morte del pontefice e più precisamente dell'assassinio del papa. I testimoni hanno infatti riferito che Villot, una volta entrato nella stanza da letto del papa, dopo aver scoperto il cadavere senza alcun imbarazzo ha effettuato alcune telefonate direttamente dal telefono del papa. ${ }^{11}$

Suor Vincenza ed entrambi i segretari del Papa morto hanno visto delle carte sia nelle mani del papa defunto che sulla scrivania papale. Dopo la partenza del cardinale Villot, tutti quei documenti oltre agli oggetti personali del Papa sono spariti. Don Diego Lorenzi ha riferito che dopo la visita di Villot, egli stesso insieme a don Magee è entrato più di una volta nella stanza del papa per ricercare quei documenti, senza trovare nulla. Inoltre, don Lorenzi che dormiva nella stanza da letto adiacente a quella del papa, ha riferito di non aver sentito suonare, in quella mattina del 29 settembre, la sveglia di Luciani alle solite ore 4,30 a.m, a differenza di tutte le altre mattine.

Durante il pontificato, il papa non ha mai dato l'impressione di soffrire problemi di salute. Al massimo appariva semplicemente stanco, ma ciò veniva addebitato all'ingente quantità di lavoro cui era tenuto. Dopo le dichiarazioni di Sinigaglia le richieste di fare l'autopsia sono state intensificate, in quanto nessun esame esterno avrebbe mai potuto avere l'attendibilità e la completezza di un'autopsia.

Adesso veniamo alla domanda principale: come è stato ucciso Giovanni Paolo I ?

La nipote del Papa Lina Petri, figlia di Antonia, sorella del pontefice, è stata la prima persona esterna al Vaticano a vedere Albino Lucani morto. La stessa riferì di aver notato che la biancheria da letto era stata tolta. Lei restò in piedi, guardando il corpo di suo zio. La testa era voltata verso la porta, e sembrava che sorridesse. Rimase in quella camera per circa venti minuti, quando si accorse che le maniche del suo abito papale erano molto sgualcite, domandandosi perché erano ridotte in tale stato.

Pare che il pontefice avesse già capito che lo attendeva una morte inevitabile. Per questo motivo rimase seduto vicino alla scrivania fino a tardi, lasciando persino la porta del suo studio aperta, per aspettare l'assassino. Con ogni probabilità Luciani aveva notato persino che il campanello d'allarme era stato disattivato. Dopo essere entrato, l'assassino si è avvicinato al Papa, l'ha preso per le maniche e portato al letto. Giovanni Paolo I probabilmente è stato soffocato con un cuscino. ${ }^{12}$ La nipote Petri ha evidenziato che sul letto non c'era niente, per cui tutte le tracce del crimine erano state sicuramente, già, rimosse prima del suo arrivo in modo da non permettere alcun sospetto sulla morte dello zio. Dal video, che riproduce la prima esposizione del pontefice defunto nella Sala Clementina, si scorgono, sul viso del pontefice defunto, delle strisce sotto gli occhi e sopra le soppraciglia. Questi segni potrebbero far pensare che l'assassino abbia messo qualche cosa sul volto del Papa, soffocandolo. Resta strano che nessuna guardia svizzera si sia accorta di nulla.

II 4 ottobre Giovanni Paolo I venne tumulato. Pioveva, come se la natura piangesse per la sua scomparsa. Papa Luciani è stato sepolto nella grotta Vaticana sotto la Basilica San Pietro, vicino alla tomba del primo papa e di molti altri pontefici romani.

Fino ad oggi il Vaticano non ha fatto niente per raccontare al mondo la verità dell'assassinio dell'ultimo papa italiano.

La storia di Papa Luciani non finisce qui, perché la gente continua ancora oggi a vederlo come un santo, a partire dai suoi innumerevoli devoti miracolati che ne chiodono a gran voce la canonizzazione.

La morte di Giovanni Paolo I, non è riuscita a cancellare la buona memoria di uno dei più illustri personaggi italiani del secolo. Molte strade e piazze in Italia portano oggi il nome di Papa Luciani. Persino la nuova università romana è stata a lui dedicata.

Dal 2003 è cominciato il processo di beatificazione di Giovanni Paolo I che tuttavia è stato rallentato dai successori non italiani. II compito del nuovo pontefice argentino Francesco I, di origine italiana, è di completare il processo di beatificazione di Giovanni Paolo I nei tempi più brevi. II Papa attuale deve finalmente dire al mondo la verità - perché e come è stato ucciso Albino Luciani, l'ultimo pontefice italiano.

\section{References}

Albino Luciani dal Veneto al mondo, Viella, Vicenza, 2010, 602pp.

Chi sarà il nuovo papa? Albino Luciani. Con i poveri (non a sinistra), «ll Giorno», 22 agosto 1978.

Il papa che ci occorre, «Panorama», 22 agosto 1978, pp.78-79

${ }^{11}$ Shkarovskiy S. 33 giorni in Vaticano, Edizioni Segno, Tavagnacco (UD), 2012, 132pp.

${ }^{12}$ Shkarovskiy S. 33 giorni in Vaticano, Edizioni Segno, Tavagnacco (UD), 2012, 132pp. 
Incitti L. Papa Luciani. Una morte sospetta, L'Airone Editrice, Roma, 2001, 125pp.

Les derniètres manouvres vaticanes, «Le Manin» (Paris), 23 agosto 1978.

Rocha L.M., La morte del papa, Cavallo di Ferro, Roma, 2006, 430pp.

Serafini L. Albino Luciani II papa del sorriso, Messaggiero di Sant'Antonio, Padova, 2008, 186pp.

Shkarovskiy S. 33 giorni in Vaticano, Edizioni Segno, Tavagnacco (UD), 2012, 132pp.

Un papabile de compromis, Albino Luciani, «Le Manin» (Paris), 23 agosto 1978.

Yallop D., In God's name, BANTAM BOOKS, London, 1984, 388pp.

http://it.wikipedia.org/wiki/lllustrissimi

http://www.religio.ru/lecsicon/03/120.html 